\title{
GRAMMATISCHES UND ETYMOLOGISCHES.
}

\section{Zu den irischen Verben vom Typus bongid, -ding.}

Nachdem Pokorny mit Recht dafür eingetreten ist, dals die $i$-Wurzeln ihren Präterital-Stamm mit $i$ reduplizierten, ${ }^{1}$ ) also auch für dedag-(for-rudedach su Ml 96 c 17), falls es zur W. dheigh-dhigh- gehört, ein älteres *didoig- vorauszusetzen ist, ist natürlich auch für die $\iota$-Wurzeln Reduplikation mit $u$ anzunehmen. Ich würde also nicht mehr, wie Handb. § 685, für con·bobig, at·bobuid, in lolaigh ('lolaid, loiligh O'Dav. 1130, AL IV 16,25) älteres *.bebig usw. ansetzen; vielmehr sind die keltischen Grundformen *bubouge, *buboude, *lulouge wie altind. bubhōja. Dagegen spricht nicht at'roebaid SR 3997; denn roe- hät sich weit über sein ursprüngliches Gebiet hinaus ausgebreitet: ad·roethach Sanctans. Hymnus 20 (falsch von mir erklärt §691, richtig von Pedersen $\S 839$ ), co rróebriss, barroebris Ériu 4, 203, ad'roegaid V.Trip. 566, 24, do'roemadair SR 7955 (do'roemaidir 2709), do'ráegart ZCP 3, 206, 3. Für cualae ist ja längst eine Grundform *kukloue angenommen, wenn auch das $i$ statt $y$ in mkymr. cigleu nicht genügend erklärt ist.

Eigentümlich ist die 3. Sg. des Futurums von bongid: nescietur cóich·biba, coich·beba 'wer siegen wird, wer sterben wird' V. Trip. 88, 1,2) da andere Verben dieser Klasse den Stammvokal abwerfen, z. B. fo lil Ériu II 208,28. Es ist wohl durch das schwache Futurum auf $-b a,-f a$ oder durch das

1) ZCP 11, $24 \mathrm{ff}$., Idg. Forsch. 35, $336 \mathrm{ff}$.

2) Gehört hierher auch for biba (so YBL, farbbiba, forbeba die anderen Hss.) sochaide (n-)inmunn de rigaib Herend Táin Bó Fraich \$15? Oder eher zu for-bond-? 
intransitive 'mema (zu maidid) beeinflufst; ${ }^{1}$ ) vgl. Subj. :conblıa AL IV 334. Regelrecht ist der Subj. to uithi-b Érin VII 162,5, cen(n)iro'taithim AL III 56, 21.

Besonders auffallend sind bekanntlich die Formen des Kompositums to-aith-bong- 'lösen, auflösen (eine Streitfrage, einen Vertrag)', 'öffnen (ein Grab)', die Pedersen bewogen haben, es ganz von bong- zu trennen (\$663. 669). Neben Formen wie in tan tathbongar O'Dav. $980=$ in tan do'nathmongar AL V 506, 10, conda'tathbongat AL II 382, 3 stehen solche wie do'aithbiuch 'abrogo' SGall. 22 b2, taidbegar V.Trip. 160, 19, taidbecti 'enodanda' Enchir. 75 a und das häufige Abstraktum taidbech taithbech, jünger taithmech. Das letztere ist leicht zu erklären; es kann lautgesetzlich entstanden sein, indem bei Synkope des Vokals der Präposition athe- (gall. ate-) die Lautgruppe thb palatalisiert wurde. So reimte das Wort mit dem gegensätzlichen cuimrech 'Binden, Fesselung' zu con rig, 1. Sg. con'riug, Pass. con:regar, und nach diesem bedeutungsverwandten Verb sind -binch, -begar usw. gebildet. Gelegentlich sprang dann das palatalisierte $b$ in andere Komposita über: debech neben debach (Contrib.), co toibget Ériu I 215, nacon'toibget AL V 254, 2.

Umgekehrt lälst sich con'utuinc con'utaing, Abstr. cumtach 'bauen' usw. aus com-üss-di(n)g-nicht lautgesetzlich erklären. Es hat hier offenbar Auschlufs an tongid stattgefunden, das in der Verbindung mit com- (cotach) gleichfalls mit $d$ anlautete.

Wie es sich mit tongid 'schwört' (kymr. tyngu bret. toui) selber verhält, ist nicht klar. Es bildet bekanntlich gleichfalls Formen wie Abstr. dithech, $\epsilon$ (i)tech; fre(i)tech, Prät. ducuitig, du'cuitich, at'guitigh (0'Dav. 39), Pass. ad'cuitecht, die scheinbar auf eine $i$-Wurzel weisen. Wenn Pedersen $\S 849$ mit Recht korn. ty, tye von bret. toui trennt, was mir zweifelhaft ist, und wenn seine bestechende Etymologie von éithech 'Meineid' als zusammengesetzt mit der untergegangenen Präp. $e(p) i$ (gr. '́ciogxos) das Richtige trifft, so mülste hier die Stammischung weiter hinaufgehen. Immerhin mufs man mit der Möglichkeit

1) Auf eine Analogiebildung in umgekehrter Richtung nodon'sel zu slaidid Fled Bricrenn $\S 48$ habe ich Handb. $\S 666$ aufmerksam gemacht. Die zu erwartende Form con sela steht RC 24, 17t $\$ 2$. Hierher auch ardon'sela Fél. 23. Juni zu ar'slaid 'verteidigt'. 
rechnen, dals auch in eithech eine Synkope vorliegt, wenn auch das erste Element (*ciui-?) dann einstweilen dunkel bleibt. Zur falschen Auflösung in dod·fongad Ml 36 a 21 tritt jetzt to foing Ériu VII 158,16; dạeben in demselben Text no'thoing 157, 15 und ausserdem to:thoing 13, do thoing 12 (richtig überliefert?).

\section{2. duduoecuster Ériu VII 146, 4 .}

In nech as'lu fuidel lochad no dudnioeaster 'einer, der trinkt, was Mäuse übrig lassen, oder es ifst' haben wir ohne Zweifel einen neuen Beleg für die Tatsache, dals $u$ zwischen schwach betonten Vokalen wenigstens im zweiten Glied von Komposita bis nach der Synkope sich gehalten hatte, vgl. Bresual, Conual (K. Meyer, Über die älteste ir. Dichtung II 4). Es ist dud·uoestar (= späterem *dud·foestar) zu lesen, ein Beweis für das hohe Alter des Poenitentials; vgl. KZ 48, 58.

\section{Zum reduplizierten Präteritum im Irischen.}

In betreff der reduplizierten Präterita, die aufser der Reduplikazion noch durch ein zweites Präteritalzeichen charakterisiert sind, bedarf meine Darstellung im Handbuch vielfacher Ergänzung, die ich zum Teil schon anderwärts angedeutet habe.

Für die Vereinigüng des $s$-Präteritums mit der Reduplikazion bei Wurzeln, die auf einen langen Vokal endigen, hatte ich $\S 677$ nur das eine Beispiel gêris- aus gegniss- zur W. gnīund das darnach gebildete sēnis- zu sni- in adru'choisséni Ml $69 \mathrm{~d}$ 4, con sēna Fiaccs Hy. 28, ni cossēna Broccans Hy. 22, con'asensat ZCP 8, 313, 31 usw: Aber das war offenbar die allgemeine Bildungsweise.

Zu rā-, ursprünglich 'rudern', dann 'sich wohin bewegen' (imm-rā. 'auf einem Schiffe fahren'): 3 . Sg. reris rerais The Voyage of Bran I $29 \S 61$, in tan reras ebenda 43,8 , imm rerce SGall. 62 b 7, Pl. ros'versat, rersad (lies -sait) Idg. Forsch., Anz. 33,35 , also Stamm rerāss.

$\mathrm{Zu}$ ad cota 'erlangt', das ich jetzt mit Pedersen zil W. $t \bar{a}$ $=$ stā- stelle (vgl. lat. instare): 1 . Sg. ad'cotadus, ud'chodados sa, 3. Sg. ad·cotedae, ad'cotade, níctade, 1. Pl. ad'cotadsam, 3. PI. 
ad'cotatsat, trissa'n-étatsut usw. (Pedersen $\S 838$ ), also Stamm tet(h)ass-.1)

$\mathrm{Zu}$ sna- 'schwimmen' ist ein Stamm senas-aus sesinassmeines Wissens bis jetzt nicht belegt. Dals er aber bestanden hat, scheint mir das Präsens con'sla 'er geht', Pl. cota slaad Imperat. colla (collaa) zum Prät. con'sela(i), cot'sela ziu erweisen an Stelle von Präs. ${ }^{*}$ con sela, Imperat. coisle (ZCP 8, 77); es wird eben nach diesem Muster geformt sein.

An diese Verben schlielst sich die ursprünglich auf $s$ endigende Wurzel an, die in bás 'Tod' vorliegt: Prät. bebais, ro'mbebe, jünger $b e b a, \mathrm{Pl}$. bebsait. ${ }^{2}$ ) Eben von diesem Verb wird die Endung -(a)e (aus -āse) in der 3. Sg. herrühren (imm:rerce, ad cotedae).

Eine eigentümliche Ausnahme bildet $l \bar{a}$ - 'setzen, legen, werfen', das zwar die prototonierte Form rale ralae bildet, aber keine Reduplikazion zeigt. Vielleicht war einst aus *lelässdurch Dissimilazion *relāss- oder *lerāss- gervorden und danu diese unregelmäIsige Form früh durch eine unreduplizierte ersetzt worden, ein Weg, den im 9.-10. Jh. bekanntlich auch $r \bar{a}$ - und $s n \bar{a}$ : eingeschlagen haben.

Scheinbar eine Mischung des alten $s$-Aorists mit der reduplizierten Bildung bietet siassair 'er sals'; 1. Sg. 'sessar Vis. M. Congl. 93, 2 neben Perf. dessid (wohl *-sesode). Vielleicht ist aber ss nur durch den Stamm siss- 'sich stellen, stehen' Prät. 'sissestar hervorgerufen an Stelle des $d d$, das man nach rofitir. erwarten mülste (*siatir). Schon früher wird die deponenziale Flexion durch dieses Muster veranlafst sein, wie umgekehrt später tarrasair (IT I 811) für tarrastar sich wohl an siassair anschlielst.

Sicherer scheint die Verbindung des $t$-Präteritums mit der Reduplikation in siacht zu saigid, das möglicherweise unmittelbar mit dem altindischen reduplizierten W Wurzelaorist zi verbinden ist, falls nicht siassair das Muster bildete. ${ }^{3}$ ) Eine

1) Gehört hierher borg Emna ro'tetha Fél. Prol. 193 zu tām 'zur Ruhe Eingehn, grofses Sterben'? Aber das Verhältnis zu decla, das auch präsentisch ist (Pedersen $\$ 702$ ), und teidm 'Pest' ist nicht klar.

2) K. Meyer, Über die älteste ir. Dichtung II 7.

${ }^{3}$ ) Die Ansetzung einer Wurzel siag-(Handb. § 678) stammte aus einer Zeit, da man das Verhältnis von 'saig 'segat noch nicht durchschaute; 
ähnliche Form ist vielleicht fiacht in Amra ConRoi: rodon fe foebra (lies foebur?) fiacht 'der für uns den Hag der Klingen bekämpfte', vg]: fuacht fiach TBC (LU) 847; da'riuchtatar meicc do'fessat hui: digal $\therefore$ 'Söhne haben Rache genommen, Enkel werden sie nehmen' Érin VI 149, 72, wohl eine alte Nebenform des häufigen fich 'kämpfte'.

\section{Der Nominativ zu córad-.}

KZ 48, 71 habe ich bemerkt, dafs der. Nominativ *ór, den K. Meyer, A Primer of Ir. Metrics 60, und Pokorny, KZ 45,72 ff. zum Acc. Sg., Nom. Pl. córaid, G. córad, córath ansetzen; keine handschriftliche Gewähr zu haben scheint. Er wäre auch bei der Grundform gall. Cauar- (kymr. cuwr korn. caur) verwunderliclr. Die wirkliche alte Form war wohl coar, cuar, vgl. coar yaile K. Meyer, Ält. ir. Dichtung I 27 Str. 4 (Hs. L), caoin cuar Zu ir. Hss. I 51, 11 (Hs. E); vielleicht auch coar-echt mo cholaind 'die Gewalttat an einem Krieger ist meine (die vor mir liegende) Leiche' Versl. II § 27. Cuar heilst einer der Söhne der Scăthäch und ein Krieger der Aife seit der ältesten Fassung von Tochmarc Emire (RC XI 448, 77. 450, 103). Nach jenem ist cles Cúair (Windisch 'T. B. C. 280 Anm. 5) benaunt (oder ungekehrt?), vgl. Dún Cuair (Hogan). Hier ist das Wort als 0 -Stamm flektiert. Ist das das Ursprüngliche, so entspricht es genau gall. Kaviaoos und hat die dentale Flexion erst im Anschlufs an $c(a) u r$ c(a)urad 'Held' angenommen, mit dem es die Schreiber oft verwechseln.

\section{Air. lour 'genug'.}

'Es genügt' heifst air. roffera, 'genug' aber lour loor. Es war also aus ro-uero- durch Dissimilazion louero- entstanden und zwar in früher Zeit, da kymr. llawer 'viel' korn. lawer, lower entspricht, 'ein nenes, sicheres Beispiel für britannisch -aun- aus -oun.

s. Handb. I 525 zu S. 50, Pedersen II $610 \mathrm{f}$. Es ist übrigens bis hente noch nicht klar, weshalb in einigen Beispielen der Übergang von $e^{i} \mathrm{zn} a^{i}$ gemeinirisch, in anderen nur mundartlich ist. 


\section{Ir. marbu 'ich töte'.}

Vor Jahren machte mich Schlutter auf einen (verderbten) irischen Zauberspruch aufmerksam, der dem Menschen oder Tier ins linke Ohr gesprochen werden soll zur.Vertreibung eines Wurms, der mit dem Wasser verschluckt worden ist. $\left.{ }^{1}\right)$ Der verständliche Anfang lautet: gono mil orgo mil marbu mil 'ich verwunde das Tier, ich schlage das Tier, ich töte das Tier'. Orgo ist absolute 1. Sg. wie tíagu, tongu. Marbu ist der erste Beleg dieser Art bei einem $a$-Verb; bisher kannte man nur die konjunkten Formen not caru Fél. Epil. 311, ar'coimtiu Ml $141 \mathrm{c} 5$. Gono kann man auch hierher zählen, da gonaid sein Präsens im allgemeinen schwach bildet. Aber die konjunkte 3. Sg. heifst immon'goin $\mathrm{Zu}$ ir. Hss. I 57, ciche goin TBC (LU) 1082, ni.gon 2390; das Verb steht also gewissermalsen auf der Grenze zwischen starker und schwacher Flexion.

\section{Ir. sunda,}

die Nebenform von sund 'hier', z. B. Wb 25 b 9, ist offenbar' als sund-sa zu deuten und zeigt, dals einst auch die Verstärkungspartikeln der Lenierung unterlagen.

\section{8. $p 1$;, $p l$ im Keltischen.}

Im Anlaut haben $p r$ - und $p l$ - ihr $p$ bekanntlich verloren; aber im Inlaut scheint mir $b r, b l(b r, b l)$ daraus geworden zu sein.

ad·cobra 'wünscht, will', Abstr. accobor accobur ist nicht von lat. cupere zu trennen, zumal dieselbe Ableitung kuproim Italischen vorliegt. Ciprum (d. i. cyprum, cuprum) sabine 'bonum' meldet Varro, De ling. lat. V 159, der damit den

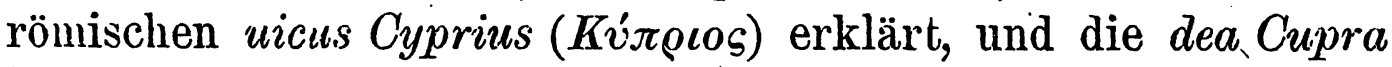
wird in Picenum verehrt, Cubrar matrer (Gen.) in Umbrien.

gabor 'caper', gabur Thes. II 360, neuir. gabhar m. 'Ziege', kymr. gafr bret. gaour, gaor f: 'Ziege', altbrit. Gabrosenti 'Ziegenpfad', gall. Gabrus Gabra, Gabrillus Gabrilla, Gabromagus sind gewils dasselbe Wort wie lat. caper capra, aisl. hafr aengl. hofer 'Ziegenbock' usw., wenn auch das $g$ - noch nicht

1) Bibl. der ags. Prosa, hgg. von Grein-Wülker VI 12ó (Nr. X). 
erklärt ist (Handb. $\$ 219$ ). Die jetzt beliebte Zurückführung von kymr. caer-iwrch 'Rehbock', air. caíra, cáera, Gen. cáerach 'Schaf' (neuir. caora f.), gall. Caeracates auf angebliches kapero(Rhys RC II $337 \mathrm{f}$., Stokes bei-Fick III ${ }^{4}$, 64) hat keinerlei Wahrscheinlichkeit. Kymr. caeriwich mit den Nebenformen cariwrch, carwiwrch scheint mir Silvan Evans s. v. mit Recht von carw 'männlicher Hirsch' abzuleiten.' Und der Name des Schafs kairāk. gehört dentlich zu air. cáer 'Klumpen, Traube', neuir. caor 'Frucht der. Eberesche'; es ist nach seinen Exkrementen benannt, die es so charakteristisch von Ziege und Rind unterscheiden.

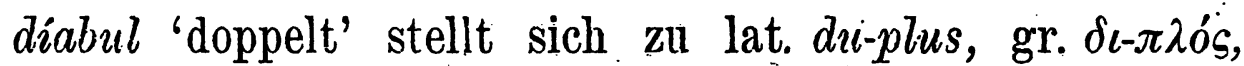
im ersten Glied am nächsten zu got. tweifl (Acc. Sg:), ahd. zuĩval 'Zweifel'.

So braucht man für *ebraid 'er wird gewähren' (zu gr.

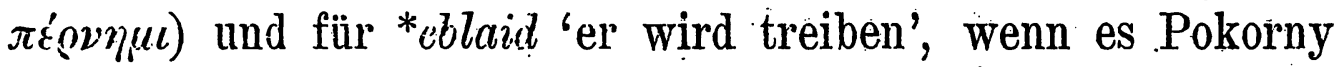
Idg. Forsch. 38, $115 \mathrm{f}$. mit Recht zu lat. pellere stellt, nicht mehr meine etwas künstliche Erklärung Handb. $\S 227$; sie sind regelrecht aus (p)iprās- und eventuell (p)iplās- entwickelt. 1) Zu lingid 'springt', das Osthoff, Morphol. Unters. 6, 23 mit gr. $\pi \lambda \iota \chi \alpha$ 's 'interfeminium', $\pi \lambda i \sigma \sigma o \mu \alpha i$ 'gehe mit ausgespreizten Beinen' verbindet, erwartet man ein Präteritum *eblaing; leblaing hat das l- von lingid usw. bezogen.

\section{Kymr. dywed = ir. do fieith.}

Ir. fethid 'er geht (fort)', Pl. fethet, Perf. ro'fáith, Pl. ro'fäthatar und do'feith 'kommt', Prät. do fāith (Handb. I 447, Idg. Forsch., Anz. 33, 36) stimmen so genau zu kymr. dywedyd 'sagen', Prät. mkymr. dywaut dywawd, dafs an der Identität nicht $\mathrm{zu}$ zweifeln ist. Der Bedeutungswandel im Kymrischen ist .allerdings auffallend. Früher wurde auch das Simplex gwedyd so gebraucht; ni wedai air 'er sprach kein Wort', celwydd gwedyd 'falsehood of speaking' zitiert Owen Pughe ${ }^{3}$ s. v. Darf man an Ausdrücke erinnern wie ir. a n-do'rigne do fertaib, ni fail dune do[a]·decha 'was sie an Wundern getan hat - es gibt keinen Menschen, der es (mit Worten) erreichte'

1) $\mathrm{Zu}$ dieser Futurbildung s. Iilg. Forsch. 38 (gedrnckt, nber noch nicht erschienen). 
Broccuns Hy. 81 oder an saigid 'zielt mit der Rede anf etwas ab' z. B. Wb 22 a 3? Lat. uetare, das wohl zu der gleichen Wur'zel gehört, bezieht sich allerdings auch aufs Sprechen, hat aber mit dem kymrischen Gebrauch keine nähere Berührung. Näher liegt diesem vielleicht die Bedeutung des schwachen irischen Verbs fethid 'richtet seine Aufmerksamkeit auf etwas, beobachtet', 2. Sg. Prät. rofethis Fél. 24. Juni, 7. Aug.; doch ist zweifelhaft, ob dieses mit dem anderen fethid zusammenhängt

Bonn.

R. Thunneysen.

\section{ALTIRISCH $E R U L B$ n. pr.m.}

Marstrander folgt in seinem 'Bidrag' S. 51 dem Vorgange von Stokes und Todd (Cog., clxxiv Anm.3), indem er diesen altir. Namen aus altn. Herioifr herleitet. So kommt er zu dem Schlusse (S. 63), dafs letzteres schon ca. 885 als Heriulf $f_{R}$ anzusetzen sei. - Aber die von ihm angeführten Daten, an welchen der Name vorkommt, beweisen etwas ganz anderes. An allen Stellen (AU 949, 958 usw.) handelt es sich nicht um eine Erulb genannte Person, sondern um einen ua hEruilb, d.h. um ein Mitglied des Stammes der $\bar{U}_{i}$ Eruilb, nicht, wie Todd und Hennessy übersetzen, 'grandson of Erulb'. Die Üi Eruilb gehörten zu Cenēl Eogain, und ihr Stammvater war Erulb mac Murchada m. Māeldūin m: Āeda Ollān. S. CZ VIII 294,33. Da sein Grolsvater Māeldūin 788 starb und sein Vater. Murchad i. J. 823 von Niall Caille seines Königssitzes in Ailech beraubt wurde, wird er etwa um 800 geboren sein, zu früh als dals er einen nordischen Taufnamen hätte erhalten können. Wir haben also Entlehnung aus altengl. Herewulf, Herulf.

1) Wenn Todd a. a. 0 . sagt 'the family of 0 'hEruilbh was of Danish origin and seated in the neighbourhood of Kildare', so hat er letzteres wöhl vorschnell aus AU 963 erschlossen, wo ein Niall ua hEruilb erwähnt wird, der mit eigenem Gelde die Mönche von Kildare von den Wikingern loskauft. Über einen anderen Ui Eruilb genannten Stamm s. Buch von Fenagh S. 390.

Berlin-Wilmersdorf.

Kuno Meyer. 